\title{
La Commission Centrale d'Ethique: digne de foi, efficace et flexible
}

\author{
M. Vallotton, Président de la CCE
}

L'Académie Suisse des Sciences Médicales, créée en 1943 par les cinq facultés de médecine, les deux facultés de médecine vétérinaire ainsi que la Fédération des médecins suisses s'est d'emblée préoccupée de problèmes d'éthique médicale et a édicté ses premières directives sur «Le diagnostic et la définition de la mort» en 1969. Devant l'ampleur et la complexité de la tâche, elle créait en 1979 une Commission Centrale d'Éthique (CCE). L'ASSM va commémorer cet événement par un symposium le 23 septembre 2004 à Berne. A l'occasion de ce jubilé, le Prof. Michel B. Vallotton de Genève, Président de la CCE, adresse un clin d'œil au passé et porte son regard vers l'avenir.

\section{Bref rappel historique}

Si la création par l'Académie Suisse des Sciences Médicales (ASSM) de la Commission Centrale d'Ethique (CCE) date bien de 1979, c'est déjà en 1969 que l'Académie édictait ses toutes premières directives sur «Le diagnostic et la définition de la mort». Devant l'ampleur de la tâche et constatant le nombre des problèmes médicaux requérant des directives médico-éthiques au cours des 10 années suivantes, l'Académie se vit devant la nécessité de confier à une commission spéciale la mission de choisir parmi ces thèmes les plus urgents à traiter, d'élaborer puis de présenter au Sénat les textes de nouvelles directives. Les présidents suivants ont conduit les travaux de la CCE: Prof. Otto Gsell 1979-1982; Prof. Bernard Courvoisier 1983-1992; Prof. Walter Hitzig 1992-1999, Prof. Michel B. Vallotton dès 1999.

\section{Evolution de la procédure de travail}

Etant donnée la complexité et le caractère spécial des sujets abordés, la CCE devait s'attacher des spécialistes des domaines traités, des éthiciens, des juristes, des délégués de l'Association Suisses des Infirmières(ers), en plus de ceux du Comité directeur de la FMH. Elle a dû même confier à des sous-commissions ad hoc la préparation des projets de textes des directives. Ces sous-commissions, formées de membres de la CCE ont été complétées d'experts des divers sujets, de représentant d'institutions (médicales ou non-médicales) ou d'associations concernées; elles ont de plus, afin de ne pas s'agrandir à l'ex- cès, saisi l'occasion de consulter d'autres experts sur des points particuliers en cas de nécessité. Suivant en cela la tradition démocratique du Pays, depuis plusieurs années les projets de directives sont dans un premier temps soumis à consultation ouverte après leur publication dans le Bulletin des Médecins Suisses et même, plus récemment, adressés aux milieux concernés pour susciter leur prise de position. Ce n'est qu'après analyse approfondie des remarques, propositions ou opinions reçues que le texte final amendé est soumis à nouveau à l'approbation de la CCE (avec des allers et retours possibles) puis du Sénat pour son approbation définitive et sa publication dans le Bulletin des Médecins Suisses, sous forme de brochure séparée ainsi que sur le site de l'Académie (y compris quelque temps plus tard en version en langue anglaise depuis deux ans). Depuis 2002, les sous-commissions ont choisi, avant l'ouverture de la consultation générale, de soumettre les projets de texte à plusieurs experts externes appelés en préconsultation pour s'assurer de leur adéquation, de leur pertinence, de leur praticabilité et de leur recevabilité.

\section{Efficacité, avantages et désavantages}

Selon ces procédures, qui peuvent paraître longues et complexes à première lecture, ce ne sont pas moins de vingt directives médicoéthiques qui ont été édictées ou révisées un total de 14 fois (de 1 à 4 fois chacune) (fig. 1 et 2). Deux directives (Procréation médicalement assistée; Stérilisation de personnes mentalement déficientes) ont pu être retirées et pour la deuxième remplacée par de simples recommandations, lorsque des lois fédérales réglant leur objet sont entrées ou en cours d'entrer en application. Ces dernières, bien que parfois avec des points d'achoppement, s'inspiraient largement des directives de l'Académie. Ce faisant, la démonstration était apportée que les directives de l'Académie jouaient un rôle précieux et apprécié en guidant le corps médical face à un vide ou des lacunes juridiques, jusqu'à ce que le corps législatif les comble. 
Toute cette procédure présente beaucoup d'avantages. Le premier avantage indiscutable réside dans le fait que les directives sont élaborées par des connaisseurs de première main des thèmes abordés, avec une expérience pratique, et non des politiciens profanes soumis aux influence partisanes ou aux groupes de pression.
Le deuxième avantage est celui de l'efficacité, le délai entre la constitution d'une sous-commission et la publication de la version finale étant de 31 à 35 mois pour les trois dernières directives, comparé à celui bien plus long de l'élaboration de nouvelles lois fédérales (fig. 2).

Figure 1

Liste des directives médico-éthiques de l'ASSM publiées depuis 1969 avec leurs successives révisions.

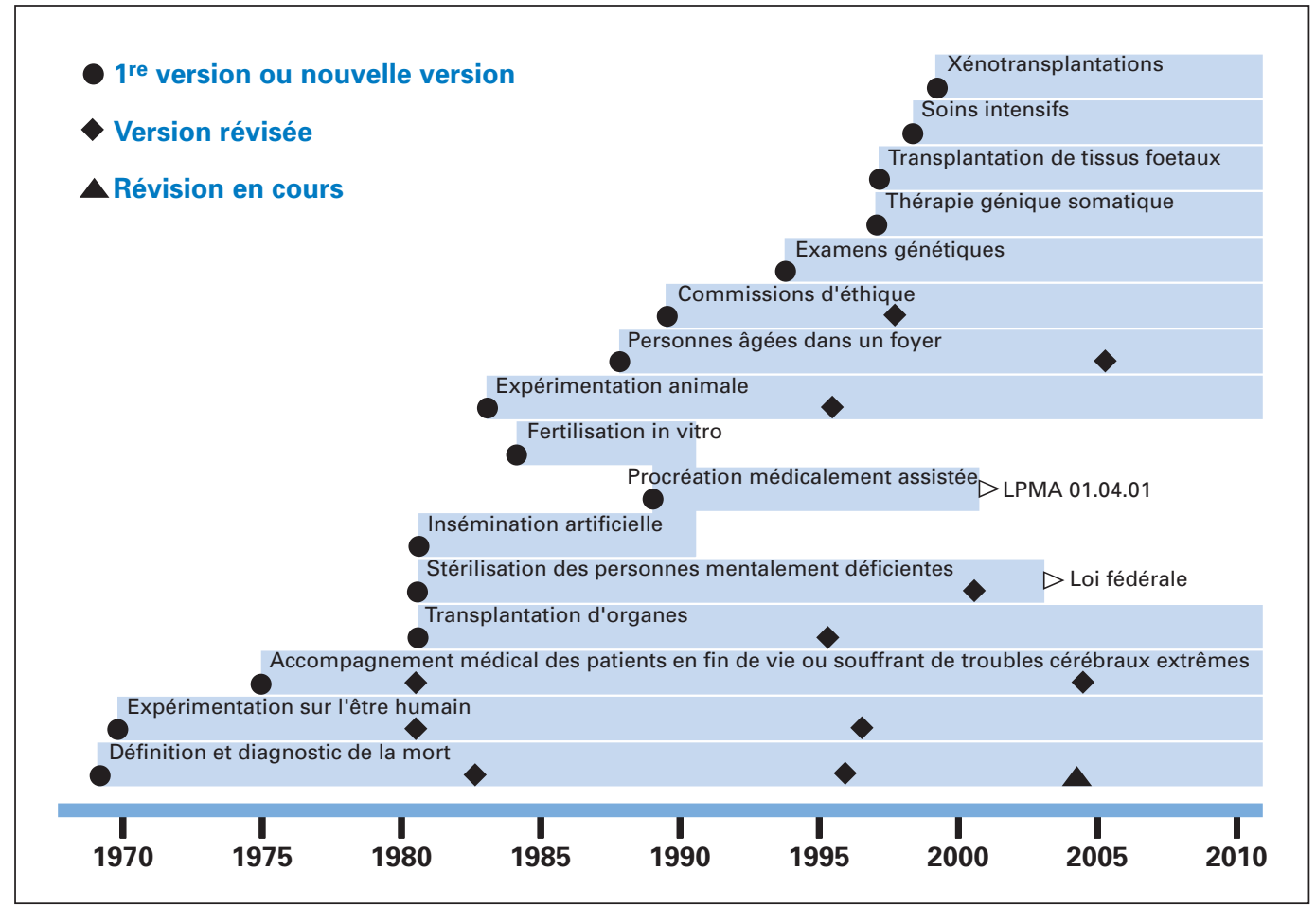

\section{Figure 2}

Liste des nouvelles directives médico-éthiques de l'ASSM publiées depuis 1998 ou en chantier. Afin de pouvoir

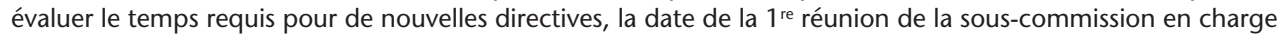
de la préparation du texte est indiquée puis celle de la publication de la version finale des directives approuvées par le Sénat après la période de consultation.

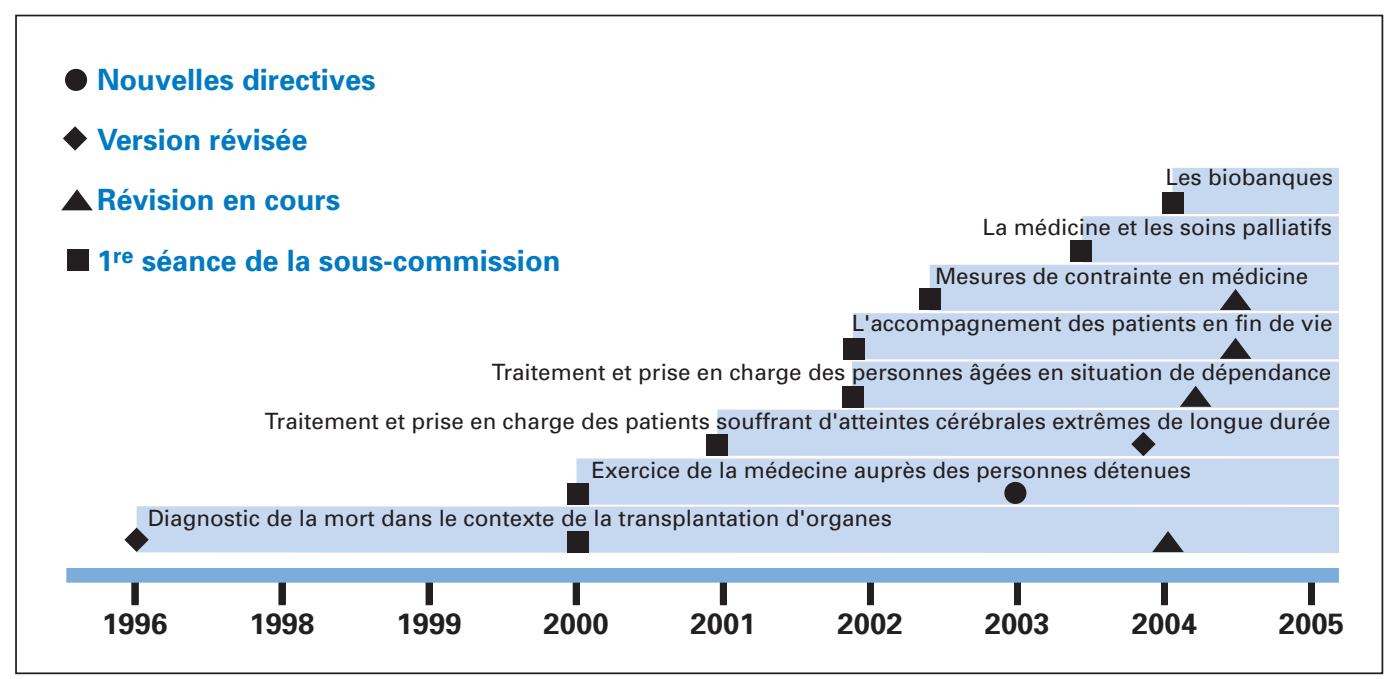


Un troisième avantage, et peut-être le principal, est celui de la flexibilité, c'est-à-dire de la capacité d'adaptation à l'évolution des connaissances en sciences bio-médicales jusqu'aux développements techniques à disposition des médecins et de leurs patients. Ces progrès et développements nouveaux soulèvent de nouveaux problèmes éthiques et requièrent des conseils pour guider les praticiens dans leur choix et décisions. A l'opposé, on connaît la difficulté de modifier une loi devenue inadaptée surtout lorsque le texte est inscrit dans la Constitution fédérale.

\section{L'autorité de I'Académie}

Les principales critiques émises par certains juristes peuvent être résumées ainsi: l'Académie au travers de ses directives ne dit pas le Droit, étant une Fondation de droit privé dont les membres sont nommés ou délégués par ses Institutions fondatrices et ne sont donc pas des élus du peuple, même indirectement.

Nous pensons que cet état de fait est justement une chance. Bénéficiant d'une autorité qui ne nous est pas conférée par décret, mais qui est reconnue à l'Académie en quelque sorte postfactum de par la reconnaissance bien informée des directives et leur acceptation libre. Bien que conscients que ces directives, même s'adressant au premier chef au corps médical, touchent, par le biais de la relation patient-médecin et du savoir technique médical, l'ensemble de la population dont les membres sont susceptibles de devenir patients, les corps législatif de plusieurs Cantons et le Tribunal Fédéral renvoient occasionnellement dans leurs lois, respectivement ses arrêts, aux directives de l'Académie. Les directives sont donc moins que des ordres (l'Académie ne disposant ni des dispositions statutaires, ni des moyens de les faire respecter) mais plus que des conseils en rendant les médecins moralement responsables de les respecter. Au demeurant, lorsque le Comité Directeur de la FMH décide d'introduire les directives dans son code de déontologie, ce devoir moral se transforme en obligation professionnelle.

L'autorité de l'Académie ne repose donc en matière médico-éthique ni sur la contrainte, ni sur la persuasion exercée du haut vers le bas, mais bien d'une reconnaissance de bas en haut exprimée tacitement par le plus grand nombre possible de ceux et celles à qui les directives s'adressent et qui ont eu auparavant l'occasion de s'exprimer à leur sujet.
D’où il découle pour tous ceux engagés dans l'élaboration puis l'approbation des directives une double responsabilité tant morale qu'intellectuelle: D'une part, la formulation et la justification des directives doivent être suffisamment univoques et éclairantes pour le plus grand nombre possible de ceux et celles à qui elles s'adressent, se sentant enclins et capables d'y adhérer (il y aura toujours aux deux extrêmes des opinions des personnes en désaccord sur un point ou un autre, avec qui il faut savoir temporiser). D'autre part, cette fois sur le plan juridique, il faut veiller à ce que les directives soient non seulement en conformité avec le Droit existant, mais tiennent compte des diverses législations cantonales ainsi que des règles et déclarations, existantes ou à l'état de projet, au niveau européen, signées ou ratifiées par la Confédération.

Ce n'est qu'en ayant toujours présent à l'esprit cette responsabilité que tous ceux et celles qui participent à l'élaboration et l'approbation des directives pourront se défendre de «privatiser le Droit». Ils trouveront une argumentation critique dans l'article [1] du Dr jur. David Rüetschi reprenant en plus condensé une publication abondamment pourvue de références [2].

\section{Nouvelles directives}

Plus immédiatement, plusieurs directives encore en chantier avant ou après la procédure de consultation seront prochainement publiées sous forme de projets (Mesures de contrainte en médecine; Banques de cellules, tissus ou organes; Les soins palliatifs) ou de version finale (La prise en charge et le traitement des personnes âgées en situation de dépendance; Prise en charge des patients en fin de vie; Définition et diagnostic de la mort en vue de transplantation d'organes). Lorsque ces divers projets auront abouti ou seront en passe de l'être, la CCE envisage d'en d'aborder d'autres qu'elle a mis à son agenda: la prise en charge et l'accompagnement des personnes souffrant d'un handicap grave; la médecine prédictive; Conditions de l'ordre de ne pas tenter de réanimation ou DNAR.

\section{Ouverture aux professions paramédicales}

Ces dernières années, la réflexion des membres des sous-commissions a porté sur des domaines impliquant pour les personnes ou les patients, au-delà des médecins, toute une équipe soignante 
formée essentiellement des infirmières(ers), des aides-infirmières(ers) et des thérapeutes de toutes spécialités intervenant sporadiquement ou régulièrement. Il en été tenu compte dans plusieurs directives récentes et recommandations. En plus des deux déléguées de l'ASI, il est veillé à ce que des représentant(e)s de ces professions soient dûment inclus ou entendus des souscommissions. Cette ouverture de l'Académie et particulièrement des sous-commissions et de la CCE vers les professions inclues dans l'expression «équipe médicale et de soins» ou d'autres équivalents a abouti pour la première fois à ce que les directives sur la prise en charge et le traitement des personnes âgées dépendants soient publiées dans le bulletin de l'ASI et soient recommandées à ses membres pour être respectées et mises en œuvre. Nous nous réjouissons de ce pas tant il correspond à la réalité du quotidien des personnes âgées dépendantes suivies de plus près et plus fréquemment par des infirmières(ers) que ce soit à domicile, en home, en EMS ou à l'hôpital. Pour les thérapeutes, vu la diversité et le nombre des spécialités et des approches, il est et sera fait appel à eux selon la spécificité des thèmes abordés.

\section{Ouverture sur l'étranger}

Un effort de visibilité et d'échange sur le plan international déjà pratiqué sera poursuivi et développé dans la mesure des possibilités et disponibilités par la participation à des réunions internationales ou à leur organisation. Déjà depuis un an les directives en langue anglaise sont accessibles sur le site de l'Académie.

\section{Evolution de l'éthique médicale}

La CCE a à prendre en compte le changement de paradigme dans la hiérarchie des valeurs éthiques représenté par l'accent mis sur l'autonomie du patient inscrite dans la Convention de Bioéthique du Conseil de l'Europe (Déclaration d'Oviedo) et inscrite dans diverses déclarations tant aux USA qu'en Europe et déjà dans certaines lois cantonales. Aussi défendable qu'elle soit, cette insistance sur la priorité de l'autonomie n'est pas encore aussi fermement revendiquée par les patients, peut-être, espérons-le du moins, par la confiance régnant encore chez nous dans la relation patient-médecin, même si certains lui reprochent un caractère paternalistique. Dans la même ligne, l'insistance sur les directives anticipées des patients comme expression de leurs désirs en matière de soins médicaux demeure un vœu rarement concrétisé tant il est encore rare que de telles directives anticipées aient été rédigées. Le débat se poursuivra sur la priorité donnée à l'autonomie par rapport au principe de bienfaisance ou de l'intérêt bien compris du patient apprécié par le corps médical. 